(31) R. M. S. Smellie, W. M. Me Indoe et J. N. Davidson. The incorporation of ${ }^{15} \mathrm{~N},{ }^{35} \mathrm{~S}$ and ${ }^{14} \mathrm{C}$ into nucleic acids and proteins of rat liver. Biochem. Biophys. Acta, 11, 559-565, 1953.

[32] G. A. Haydar, F. Chernigoy, A. Khatchadourian et S. E. Kerr. Incorporation of $\mathrm{C}^{\mathbf{1 4}}$ labeled glycine and formate by the acidsoluble guanine and adenine nucleotides of rat liver. $J$. Biol. chem., 221, 707-712, 1957.

[33] S. E. Kerr et F. Chernigoy. On the biosynthesis of ribonucleic acid purines and their interconversion in yeast. J. Biol. Chem., 200, 887-894, 1953.

[34] A. J. Tomisek, H. J. Keliy et H. E. Skipper. Chromatographic studies of purine metabolism. I. The effect of azaserine on purine biosynthesis in E. coli using various $\mathrm{C}^{\mathbf{1 4}}$ labeled precursors. Arch. Biochem. Biophys., 64, 437-455, 1956.

[35] A. R. Patton et E. G. Hill. Inactivation of nutrients by heating with glucose. Science, 107, 68-69, 1948.

[36] R. A. Mc Altister. The inactivation of certain nutrients in eulture media by heating with glucose and its relation to the growth of certain lactobacilli. J. med. lab. Tech., 12, 74-79, 1954.

[37] C. E. Lankford, J. M. Ravel et H. H. Ramsey. The effect of glucose and heat sterilization on bacterial assimilation of cystine. Appl. Microbiol., 5, 65-69, 1957.

[38] J. E. Auclatr. Studies on the antibacterial properties of cow's milk. Ph. D. Thesis, University of Reading, 1953.

[39] R. A. Finkelstein et C. E. Lankford. A bacteriotoxic substance in autoclaved culture media containing glucose and phosphate. Appl. Microbiol., 5, 74-79, 1957.

\title{
LA PRODUCTION LAITIËRE FRANCAISE DANS LE MARCHÉ COMMUN
}

\author{
par
}

A. des COURTILS

$\mathrm{Au}$ moment où nous vivons les premiers mois d'application du Traité de Rome, il paraît très difficile de situer exactement comment se présentera le marché laitier français dans le Marché Commun et quelle sera l'évolution économique de ce secteur sous l'influence des conditions nouvelles créées au travers des différentes étapes de sa mise en fonctionnement.

Le problème est complexe. C'est pourquoi je me limiterai aujourd'hui à l'examen rapide de ses principaux éléments. 


\section{Disparité des prix des produits laitiers entre les six pays}

Les trois tableaux suivants donnent un exemple des comparaisons qui peuvent être faites au niveau de la production.

TABLEAU I

PRIX MOYEN PERĢ PAR L'AGRIGULTEUR PAR KILOGRAMME DE LAIT A 37 GRAMMES DE MATIÈRE GRASSE DÉPART FERME

\begin{tabular}{l|c|c|c|c|c}
\hline \hline & Allemagne & France & Italie & Luxembg. & Pays-Bas \\
& & & & & \\
Prix en francs $\ldots$ & 33,90 & 27,90 & 27,90 & 33,90 & 31,30 \\
Prix français $=100$ & 124 & 100 & 103 & 124 & 115 \\
\hline \hline
\end{tabular}

TABLEAU II

PRIX DES PRODUITS LAITIERS

\begin{tabular}{|c|c|c|c|c|}
\hline & Allemagne & France & Luxembg. & Pays-Bas \\
\hline $\begin{array}{l}\text { Lait de consommation } 30 \mathrm{~g} \text {. } \\
\text { (francs) } \ldots \ldots \ldots \ldots \ldots \ldots \ldots\end{array}$ & 47,7 & 46,5 & 53,85 & 47,2 (1) \\
\hline $\begin{array}{l}\text { M.G. en bouteilles, prix de } \\
\text { détail (prix français }=100 \text { ) }\end{array}$ & 103 & 100 & 116 & 102 \\
\hline Francs $\quad \ldots \ldots \ldots \ldots \ldots \ldots$ & 963 & 755 & $705(2)$ & 514 \\
\hline $\begin{array}{l}\text { Beurre pasteurisé en vrac, prix } \\
\text { de détail (prix français }= \\
100) \ldots \ldots \ldots \ldots \ldots \ldots \ldots \ldots\end{array}$ & 92 & 100 & 93 & 68 \\
\hline $\begin{array}{l}\text { Francs } \ldots \ldots \ldots \ldots \ldots \ldots \\
\text { Edam } 40 \% \text { prix de gros (prix } \\
\quad \text { français }=100) \ldots \ldots \ldots \ldots\end{array}$ & 312 & 369 & & 210 \\
\hline
\end{tabular}

Lorsqu'on examine les chiffres, on s'aperçoit très rapidement que si la constatation des prix est relativement aisée sur les marchés de gros, elle devient déjà plus délicate au niveau du producteur de lait et présente des difficultés certaines au stade de la transformation.

Les études en cours tentent d'apprécier l'amplitude de ces disparités tout au long de la chaîne laitière : mais il convient de souligner qu'il s'agit plus d'une tentative que d'une appréciation exacte,

(1) Livraison à domicile.

(2) En plaquettes. 
toute suggestion étant a priori sujette à caution dans ce domaine et pour plusieurs raisons parmi lesquelles on peut citer :

- la nécessité de travailler sur des moyennes dont on n'est jamais sûr qu'elles sont établies de façon comparable ;

- la difficulté de déterminer un coût représentatif de la transformation. Les prix de revient sont variables d'une usine à une

TABLEAU III

PRIX DÉPART FERME PAR, KILOGRAMME DE LAIT A 37 GRAMMES DF MATIẺRE GRASSE

\begin{tabular}{|c|c|c|c|c|c|}
\hline & Allemagne & France & Italie & Luxembg. & Pays-Bas \\
\hline a) Prix perçu par & & & & & \\
\hline l'agriculteur .... & 33,90 & 27,20 & 27,90 & 33,90 & 31,30 \\
\hline $\begin{array}{l}\text { b) Subvention (taux } \\
\text { moyen) } \ldots \ldots \ldots\end{array}$ & 2,70 & 0 & 0 & 9,40 & 4,40 \\
\hline $\begin{array}{c}\text { Prix sans subven- } \\
\text { tion }(a-b) \ldots \ldots\end{array}$ & 31,20 & 27,20 & 27,90 & 24,50 & 26,90 \\
\hline $\begin{array}{c}\text { Prix }(a-b) \text { français } \\
=100 \ldots \ldots \ldots\end{array}$ & 115 & 100 & 103 & 90 & 98 \\
\hline
\end{tabular}

autre dans un même pays et la complexité de la détermination du coût moyen est plus grande dans l'industrie laitière que dans beaucoup d'autres secteurs. La spécialisation des usines n'est pas toujours la règle, la fabrication d'un produit s'insérant souvent dans un ensemble dont la structure varie d'un pays à l'autre ou d'une entreprise à l'autre. Par exemple le beurre est pour beaucoup d'entreprises un sous-produit du lait de consommation aux PaysBas, de la fromagerie en Italie et par contre en France il peut être une production principale associée à celle de la poudre de lait, de la caséine ou accessoire pour celle du fromage ou du lait de consommation, etc...

En admettant que ces difficultés puissent être surmontées, la comparaison des coûts moyens de production entre les différents pays présente de réelles difficultés : l'autre ;

- le matériel n'est pas absolument comparable d'un pays à

- le même mot couvre parfois des qualifications différentes, notamment en matière de salaires et de charges sociales. 


\section{Les niveaux des prix dans les six pays}

La comparaison des prix perçus par les agriculteurs, toutes primes et subventions comprises, montre (tableau I) que la France est le pays où le lait à la production est le meilleur marché. Cette constatation peut surprendre quand on connaît les différences de prix existant au niveau des produits finis, stade auquel notre pays se présente avec les prix les plus élevés pour beaucoup de produits laitiers.

On peut évidemment mettre en lumière plusieurs faits qui expliquent cette contradiction apparente.

Par exemple les subventions directes accordées aux producteurs de lait dans plusieurs pays constituent un premier élément générateur du renversement de la position respective des six pays entre le producteur et le consommateur. Il est à remarquer que tout le lait ne bénéficie pas de subventions. Souvent certaines conditions de qualité sont à remplir par le producteur. Quelquefois, seules certaines destinations sont admises au bénéfice du versement. On voit par exemple que le jeu des subventions peut permettre au transformateur hollandais de disposer d'une matière première à un prix légèrement inférieur à celui payé par son collègue français (tableau III).

Chez la plupart de nos partenaires on constate des variations importantes du prix en fonction de la destination. Tantôt la différence de rentabilité existant entre les secteurs est répercutée au niveau du producteur (Italie), tantôt elle fait l'objet d'une péréquation : le secteur à rentabilité forte est alors astreint à un prélèvement au profit du secteur à rentabilité faible (Allemagne -Pays-Bas).

Les frais de ramassage sont très différents d'un pays à l'autre. La France semble battre tous les records dans ce domaine; mais en fait les différences sont, dans certains cas, moins importantes qu'elles n'apparaissent au premier moment au travers des chiffres. Il y a à cela plusieurs raisons : par exemple en Allemagne et aux Pays-Bas, le lait est généralement payé aux producteurs "rendu quai usine ", les frais de ramassage étant déduits sur la feuille de paie mais à un taux qui ne correspond pas nécessairement au prix de revient de l'opération et peut lui être inférieur.

De ces différentes façons d'opérer, il ressort que, dans bien des cas, si le prix payé aux producteurs est relativement bas dans notre pays, l'usine française se trouve souvent payer en fin de compte la matière première plus cher que ne le font les usines de nos partenaires. 
Si nous examinons les frais de transformation, nous voyons qu'ils apparaissent nettement inférieurs chez nos partenaires. La comparaison des frais totaux en usine fait ressortir que les coûts français sont presque le double de ceux des Pays-Bas, par exemple. Par contre au niveau de la distribution, nos partenaires se montrent sensiblement à notre niveau.

Pour le beurre, la disparité des frais de fabrication paraît moindre, mais quand on regroupe les frais de ramassage et de fabrication, on aboutit à des frais totaux usine qui sont encore presque le double de ceux des Pays-Bas et d'un tiers supérieur à ceux de l'Allemagne. Les frais de distribution sont également plus élevés en France (exception faite de l'Allemagne).

Pour la poudre de lait écrémé Hatmaker, on retrouve le même décalage entre la France et les Pays-Bas. Par contre, l'Allemagne se montre plus chère que nous.

Les prix à la consommation reflètent l'incidence des divers facteurs qui jouent dans le sens d'un abaissement des coûts. Les subventions accordées à la consommation accentuent parfois le décalage (Luxembourg : forte subvention au beurre, Pays-Bas : subvention au lait de consommation).

En résumé, quand on étudie les causes de disparité, le problème se présente sous un jour particulier pour chaque produit laitier bien que le fond du problème soit commun à tous.

Si nous prenons comme exemple le cas du beurre hollandais par rapport au beurre français, on peut estimer que les principales causes de disparité sont:

- la subvention versée à chaque producteur par l'Etat en plus de la partie du prix payée par le collecteur ;

- la péréquation des prix, le marché du beurre étant soutenu par un prélèvement fait sur le lait de consommation ;

- les facteurs intervenant dans le ramassage qui sont souvent plus bas chez nos partenaires (salaires, essence, amortissement des camions). Il faut souligner, par exemple, qu'en France, la dépense de carburant représente environ $30 \%$ du prix de revient du lait ramassé. Or, aux Pays-Bas, le prix du fuel est égal à $29 \%$ du prix français et celui de l'essence à $56 \%$.

- la structure des zones de ramassage avec, au premier rang, la densité laitière ? Signalons à cet égard qu'en Allemagne, aux Pays-Bas, en Luxembourg, pays où les frais de ramassage sont les plus bas, chaque entreprise dispose dans la plupart des cas d'une zone exclusive de ramassage ;

- la répartition du prix du lait entre matière azotée et matière grasse joue aussi un grand rôle. 


\section{L'harmonisation des prix}

C'est là le problème essentiel, à la fois complexe et délicat, qui devra être réglé au fur et à mesure de l'entrée en vigueur des règles du Marché Commun.

Une première conclusion paraît d'ores et déjà certaine. Du côté français, les prix payés aux producteurs paraissent compétitifs. Mais il est à remarquer que le producteur français dispose de facteurs de production souvent plus élevés qu'à l'étranger. Il serait donc souhaitable que l'application du Marché Commun dans l'ensemble des secteurs économiques entraîne une diminution des coûts des moyens de production pour l'agriculteur français.

D'autre part, les charges qui pèsent sur le transformateur français placent ce dernier en mauvaise posture par rapport à ses concurrents étrangers.

Le développement de la concurrence sera de toute façon impuissant à réaliser l'harmonisation des coûts à l'intérieur du Marché Commun. La coordination des politiques sociales apparaît au moins aussi importante quand on constate l'incidence sur les prix de revient des charges sociales et de l'égalité des salaires masculins et féminins. La coordination des politiques fiscales jouera également un grand rôle.

Mais la coordination des politiques laitières me semble être le principal objectif sur lequel doivent converger les efforts.

Dans les instances internationales, il arrive souvent que l'on reproche à la France son protectionnisme et sa politique de subvention à l'exportation. Or, sur ce terrain, la plupart de nos partenaires n'ont rien à nous envier. C'est là un problème essentiel à régler.

Il semble que nous pouvons conclure en disant que la France aborde le problème laitier du Marché commun dans des conditions qui n'apparaissent pas trop mauvaises. Mais d'immenses problèmes sont à régler : problèmes de structure, problèmes techniques, problèmes économiques, voire problèmes sociaux dont beaucoup ne ressortissent pas seulement au secteur laitier et devront être réglés à l'échelon politique.

Ce n'est qu'ensuite qu'une concurrence loyale pourra s'établir sur l'ensemble du Marché Commun.

L'adaptation progressive aux conditions du marché semble à cet égard préférable à une planification même partielle.

(C. R. Acad. Agr. 1959, n० 10, 483) 